\title{
Mechanism elucidation of Stepwise dehydration of pharmaceutical hydrate crystals by structure determination from powder diffraction data
}

\author{
Hidehiro Uekusa, Akiko Egami, Hironaga Oyama, Akiko Sekine \\ Department of Chemistry, School of Science, Tokyo Institute of Technology, Japan \\ uekusa@chem.titech.ac.jp
}

In the pharmaceutical crystal, hydration/dehydration phase transitions are often observed phenomena during manufacturing or storage. They lead the substantial crystal structure change, so they are critical for the important physicochemical properties that depend on the crystal structure, such as stability, solubility, and bioavailability. However, after dehydration, single-crystal integrity tends to degrade, resulting in powdery crystals. We have successfully revealed solid-state structural rearrangements using ab initio Structure Determination from Powder X-ray Diffraction data (SDPD) technique [1-4]. Interestingly, some crystals show "isomorphic desolvation," in which the XRD pattern does not change significantly after dehydration, meaning the initial molecular arrangement is well preserved. We can reveal an isomorphic desolvation mechanism by comparing the crystal structures from powdery crystals in the hydration/dehydration phase transitions, which can be achieved using the SDPS technique.

Carbazochrome sodium sulfonate trihydrate, a hemostatic agent, undergoes stepwise dehydration by humidity control or heating. The hydration number decreased from 3 to 2.5, 2, 1, and anhydrous form I under dry condition, and it showed isomorphic desolvation (Fig. 1). Their crystal structures were analysed by SDPD technique to show the API molecules are linked through Na cations to form polymeric structure, and the molecular arrangements were very similar. It is noteworthy that the first dehydration did not occur at noncoordinated crystalline water $\mathrm{C}$, but water molecules $\mathrm{A}$ and $\mathrm{B}$ which coordinated to Na cation were dehydrated in sequence. This removal order was explained by the crystal structures' stability after each dehydration, calculated using CASTEP quantum mechanics calculations. Even after dehydration, the molecular arrangements were almost kept by adjusting the molecular positions slightly. After removing crystalline water $\mathrm{C}$, the crystallinity degraded significantly, indicating the molecule $\mathrm{C}$ is essential for stabilizing the whole crystal structure. Thus, the mechanism of the stepwise dehydration behaviour, and isomorphic desolvation were revealed by SDPD technique.
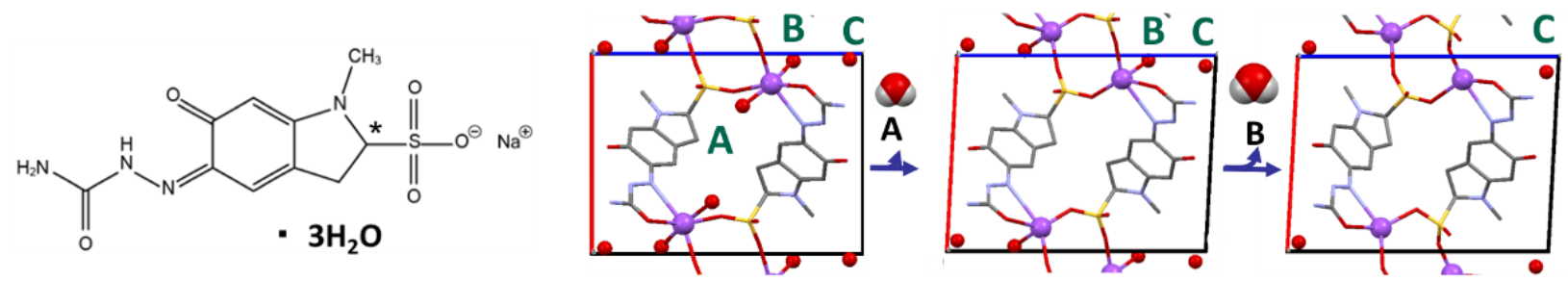

Figure 1. Chemical diagram and molecular arrangement of trihydrate, dihydrate, and monohydrate phases of Carbazochrome sodium sulfonate.

1. Fujii, K., et al. (2010) J. Phys. Chem. C 114, 580.

2. Fujii, K., Uekusa H., Itoda N., Yonemochi E. \& Terada K. (2012) Cryst. Growth Des. 12, 6165.

3. Fujii, K., Aoki, M. \& Uekusa, H. (2013) Cryst. Growth Des. 13, 2060.

4. Putra, O. D., Yonemochi, E., Pettersen A. \& Uekusa H. (2020) CrystEngComm 22, 7272.

Keywords: Structure Determination from Powder X-ray Diffraction data; dehydration; crystal structure, pharmaceutics; quantum mechanics

Part of this work was supported by JSPS KAKENHI Grant Number JP18H04504 and $20 H 04661$ (HU). 